\title{
Mitigation of Turbine Vane Shock Waves Through Trailing Edge Cooling
}

\author{
Bora O. Cakir ${ }^{1, *}$ Bayindir H. Saracoglu ${ }^{1}$ \\ ${ }^{1}$ von Karman Institute for Fluid Dynamics, Chaussée de Waterloo 72, B-1640, Rhode-Saint-Genèse, \\ Belgium
}

\begin{abstract}
The immense market demand on the high efficiency and lightweight aero-engines results in designs with compact high-pressure turbine stages experiencing supersonic flow field. In supersonic turbines, shocks appear at the vane trailing edges. The interaction of these shock with the neighbouring airfoils and blades on the adjacent rotor row and consequently create considerable amount of losses on the aerodynamic performance of the turbine. Moreover, periodic excitation created by the interaction of the shock waves and the motion of the turbine rotor causes fatigue problems and reduces the lifetime of the engine. Current study aims to alter the vane shock waves through blowing at the trailing edge. In order to characterize the effect of active blowing on the trailing edge flow field, a series of URANS simulations were conducted on OpenFOAM solver platform. Various blowing schemes were simulated over a simplified trailing edge geometry exposed in supersonic flow. The computations were compared in terms of shock intensity, oscillation frequency and exerted pressure forcing over the downstream components. The results showed that unsteady trailing edge blowing were able to modify the fluctuations observed on the shocks by altering the shock intensity, angle and frequency of oscillations. The classification of the wake unsteadiness, i.e. vortex shedding, in terms of trailing edge characteristics were also accomplished through frequency domain analysis of simulations.
\end{abstract}

\section{Introduction}

Thermodynamic efficiency is one of the defining factors for performance of gas turbines. It is directly proportional to the maximum attainable combustion chamber temperature. That can be explained as the reasoning behind the trend that leads working gas temperatures in high pressure turbines exceeding melting point of the blade materials [1]. Therefore, as the materials resistance in terms of withstanding extreme temperatures is considered, usage of thick trailing edges becomes compulsory. Thus, losses originating from viscous affects in boundary-layers due to interactions with blade surfaces and shock waves generated over the surfaces at the trailing edges of the blades introduce additional aerodynamic inefficiencies due to usage of finite trailing edge thicknesses which influences the overall performance of the high-pressure turbines significantly $[2,3]$.

*B Bora O. Cakir : bora.orcun.cakir@vki.ac.be 
In addition to the aerodynamic inefficiencies introduced, structural integrity of the parts is exposed to intense mechanical and thermal fatigue due to the shock waves formed. Impingement of trailing-edge shocks generated at nozzle vanes causes heat transfer oscillations over the surface of rotor blades [4], which leads to stress variations originated by inhomogeneous and transient temperature distributions over the blade geometries referred as airfoil load shakedown [5]. Also, mechanical fatigue caused by unsteady flow structures is a dominant factor determining the life time of turbomachinery parts [6]. Hence, transient variations of high intensity shock waves correspond to oscillations of extreme localized stresses on rotor blades of about 30 40\% of the mean level [7].

The flow filed at the trailing edge of a supersonic turbine vane is dictated by two shocks propagating downstream separated by the wake initiated through a field of low momentum. There is a constant pressure recirculation area named as the based region whose attributes dominate the unsteady pressure and temperature field downstream of the trailing edge. Thus, properties of base region highly influence the degree of compression, across the shock waves [8]. Moreover, the flow over the suction side of the neighbouring vane and its pressure distribution will be effected due to the propagation of one of these shocks while the other impinges on the downstream blades. Unsteady vane wake propagates downstream of the confluence region and creates further disturbance on the rotor blades [9].

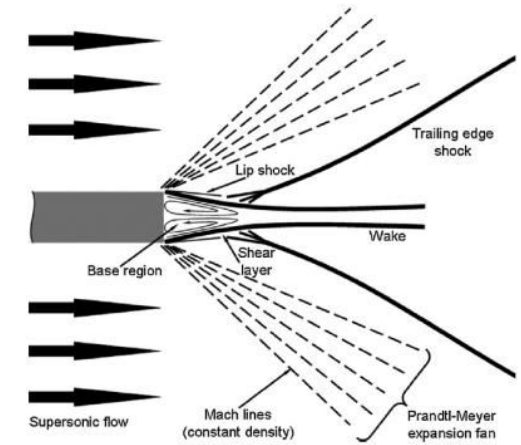

(")

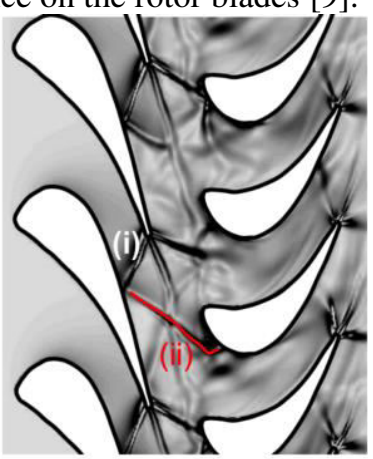

(\$)

Fig. 1. (a) Topology of supersonic flow over blunt trailing edge [17] and (b) Shock progression through stage for different time instants with respect to rotor passing period [10].

In high-pressure turbine blades, application of coolant ejection is used to protect the airfoil from immersed head load. In most practical applications, coolant flow is ejected from the blade trailing edge directly to the base region where the pressure level is an indicator of profile losses, while the shock system generates losses mainly through the shock/boundary layer interaction on the neighbouring airfoils [11].The effect of cooling applications on the loss mechanisms and the improvements obtained by employing various configurations are well documented by many experimental and numerical investigations [12-15]. Moreover, different configurations of coolant ejection geometries were tested in terms of thermal and aerodynamic efficiencies [16]. Even in cases where low blowing rates of coolant ejection are applied, significant base pressure recovery and reduction is shock intensities are obtained [17]. In addition to studies focused on enhancements attained by continuous blowing, pulsating coolant ejection sequences also showed promising results of decreasing the shock intensities at the trailing edge of the turbine blades where relevant decrease in the shock intensity with respect to a continuous blowing can be attained $[18,19]$. 


\section{Methodology}

\subsection{Numerical tool}

For the simulations, the numerical solutions are performed by OpenFOAM CFD package with pressure based solver sonicFOAM. The $k-\omega$ turbulence model was chosen for the turbulence closure problem due to its superiority in high speed flows [20]. Supersonic flow over backward facing step(BFS) was used for the numerical validation of the CFD solver owing to the analogy between the flow topologies. The validation process was performed based on the experimental results attained by Smith [21]. For the solution domain, the geometry used in the Smith's experiments was constructed in 2D. Furthermore, the boundary conditions were set as follows in order to match the conditions which Smith's experiments were conducted accordingly. The inlet static pressure and static temperature are calculated using isentropic relations where the freestream Mach number was taken as 2.5. The obtained static temperature and pressure at the inlet were $153 \mathrm{~K}$ and $7464 \mathrm{~Pa}$ respectively. The numerical solutions for the validation case was performed using a structured grid. The no-slip velocity boundary condition was applied to the adiabatic walls of the BFS. Simulation was run until all residuals reached $10^{-4}$ which was selected as the converge criteria. The static pressure values over the wall downstream of the step were normalized with freestream static pressure. Fig.2 shows the experimental and numerical results were quite strong agreement in terms of both pressure recovery and flow topology is present.

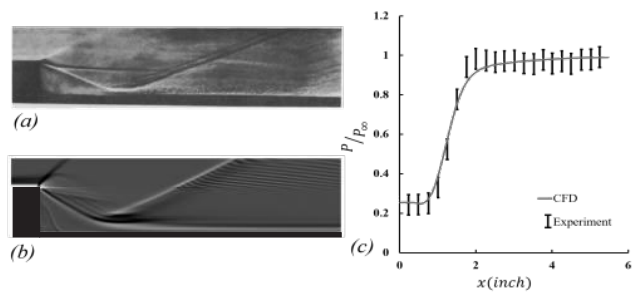

Fig. 2. (a) Experimental shadowgraph obtained by Smith, (b) Numerical shadowgraph obtained from CFD, (c) Experimental and numerical pressure ratio vs distance from backward step

\subsection{Computational domain \& mesh}

A trailing edge model with a cooling slot was numerically simulated. The length of the model was $12.65 \times t$ where $t$ represents the trailing edge thickness. The cooling slot was $2.5 \times t$ long and $0.3 \times t$ wide. Cooling was admitted to a plenum from an inlet $5 \times t$ away from the trailing edge. The solution domain of the model was enclosed by $21.75 \times t$ long and $20 \times t$ wide boundaries. In order to increase the resolution of boundary a structured mesh with $y^{+}<1$ was created over the model. Triangular elements with minimum face size of $0.025 \times t$ was used on the free stream. The resultant mesh had 238544 hybrid elements. Simulations were performed at free-stream Mach number of 1.2 and Reynolds number of $4 \times 10^{6}$. The static temperature and the static pressure of the initial flow field were set to $255 \mathrm{~K}$ and 100 $\mathrm{kPa}$ respectively. The $2 \mathrm{D}$ model used for the simulations was represented as the trailing edge of a full-length turbine blade section which also used for experimental investigation of the use of coolant ejection to control base pressure and shock generation [19]. Hence, a boundary thickness of $9.7828 \times 10^{-3} \mathrm{~m}$ at the starting point of the model is defined in order to account for upstream effects [22]. Three modes of coolant blowing were simulated; no blowing, steady and pulsating blowing. For coolant blowing three pressure levels were tested; $110,150,190 \mathrm{kPa}$. Fluctuations on coolant blowing was created at $200 \mathrm{~Hz}$ with an amplitude of $20 \%$ of mean level. All flow attributes analysed for blowing cases were 
normalized with the corresponding values at no blowing conditions which was set to be the baseline. The temporal evolutions were analysed with respect to the pulsation period of $T=5 m s$.
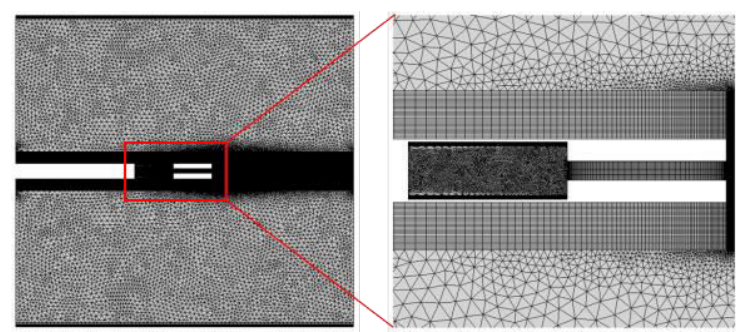

Fig. 3. Computational domain of single slot purge geometry

\subsection{Method of analysis}

In order to analyse the variations on the major flow field structures, a series of integration lines were created inside the computational domain. For the determination of base region properties, flow properties over the lines at the backward faces of the trailing edge were used as shown in Fig. 4-a. Mass flow rate through the base pressure were constructed by averaging the data over the line which was located at the middle section of the purge channel as illustrated in Fig. 4-a.
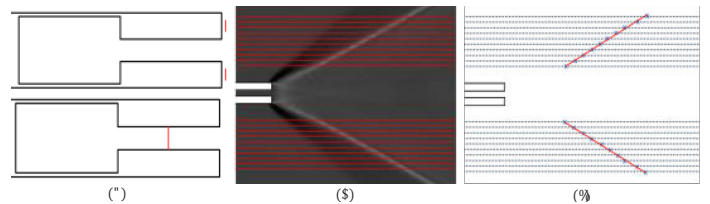

Fig. 4. (a) Data extraction lines at purge channel, cooling cavity and base region, (b) shock data extraction and (c) shock reconstruction

\subsection{Forcing analysis}

Right wing shock generated at vane trailing edge propagate downstream and impact to the leading edge of the rotor blades (Fig.5-a). Thus, an intense static pressure variation over the rotor crown is created. This interaction induces strong fluctuations on pressure forces over the rotor and additionally may create fatigue issues on the turbine structure. In order to understand the effect of active cooling over the rotor forcing, the unsteady static pressure variation across the right running shock wave (Fig. 5-d) was extracted over an interrogation plane located at a distance scaled to stator/rotor distance of BRITE turbine [7] (Fig. 5-b,c). The pressure increase across the shock wave was recorded with a temporal resolution of $10^{-4} \mathrm{~s}$ to quantify the unsteady forcing.
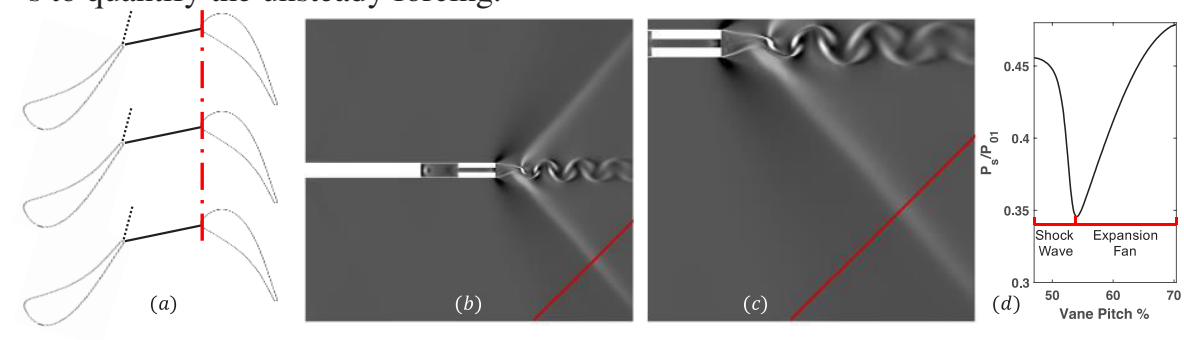

Fig.5.(a) Shock layout through turbine cascade, (b,c) Forcing analysis data extraction, (d) Forcing line topology distribution 


\section{Results}

The flow field around the trailing edge exposed to supersonic flow shows peculiar flow features. Fig. 6-a shows the baseline case where no coolant is blown. Supersonic flow upstream of the trailing edge expands through the expansion fan and deflects towards the symmetry axis of the trailing edge while forming the shear layer on each side. A von Karman type vortex street sheds from the point of confluence at a frequency of $5 \mathrm{kHz}$. This phenomenon is the main source of the wake unsteadiness and base pressure fluctuations varying $10.8 \%$ around mean level, 90 degrees out of phase on each side of the trailing edge (Fig. 6-b). Trailing edge shock decelerating and diverting the flow exert strong static pressure jump in the flow at the same frequency.
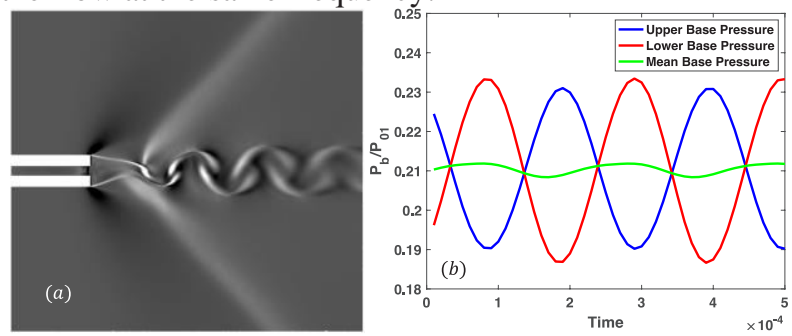

Fig. 6. (a) Numerical schlieren close-up around trailing edge and (b) variation of base pressure ratio and shock intensity with respect to time for no blowing

The effect of active cooling on the flow structures was analysed for various blowing rates. Fig. 7-a shows base pressure unsteadiness along with the cooling mass flow rate variations at three cooling rates. The pressure level at the coolant inlet was incrementally increased to supply higher mass flow rates. However, the effect of unsteadiness in pressure on the resultant coolant mass flow was dumped due to chocking of the flow at the cooling channel (Fig. 7-a). The mean base pressure level has increased for all blowing rates as compared to the baseline case. Consequently, the base pressure losses reduce. The highest augmentation was reached by the lowest blowing. The results also show that the base pressure fluctuations were modulated at the pulsation frequency for all blowing pressures.

Fig. 8 clearly represents the effect of blowing scheme on the variation of shock attributes. The shock intensity was immensely reduced by low blowing rates for both steady and pulsating ejection in exchange of high variability ( $\sim 15 \%$ of no blowing value). The effect on the average intensity and variability was progressively damped by increased blowing rate (Fig. 8-a, b, c). Similar effects are observed on the shock angle variation. Fig. 8 -d shows how widely low blowing rates increases the shock oscillations whereas the medium and high blowing rates results in similar variation in shock angle. This is due to the inertia of the fluid inside the cooling plenum damping applied pulsation at the trailing edge and consequently converging the effects towards the steady blowing conditions. Hence, using pulsating cooling above certain blowing rates for a given trailing edge geometry may not result in unsteady modulation, but rather causes stabilization of the shocks.
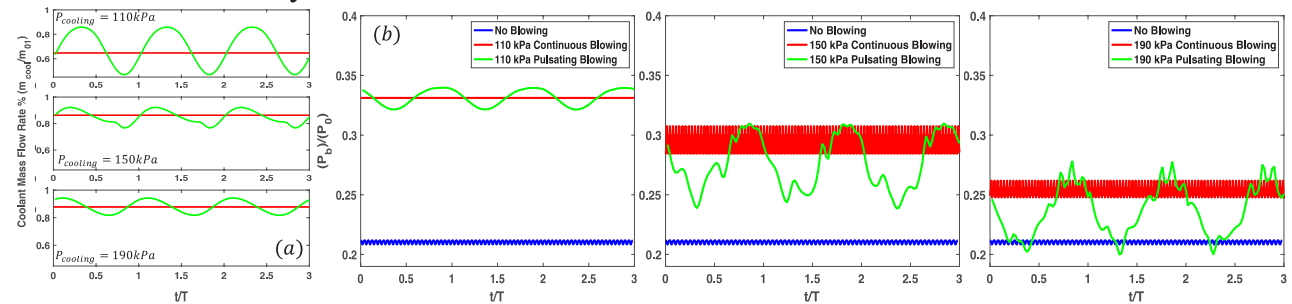

Fig. 7. Variations of purge mass flow and unsteady base pressure for steady and pulsating blowing 

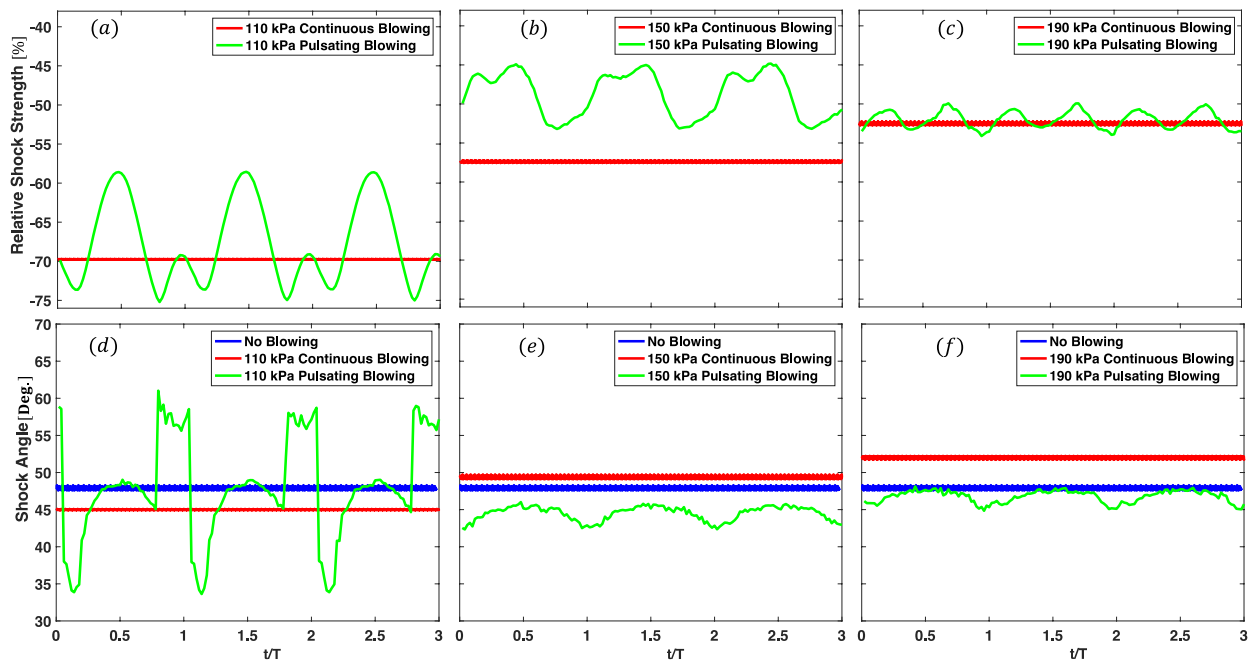

Fig. 8. Variations of shock intensity and shock angle with respect to time for steady and pulsating blowing.

Changes in pressure force exerted on the leading of rotor blades by the trailing edge shock waves can be interpreted through the static pressure variation across the right running shock wave (Fig. 9). This shock periodically impacts on the rotor crown and results in unsteady forcing on the rotor. Lowest pressure level close to the rotor is dictated by the expansion fan while the sudden increase is a function of trailing edge strength. Both flow features highly depend on the state of base region. Therefore, the resultant rotor forcing is a function of trailing edge cooling scheme. Fig. 10-a shows how the pressure jump is reduced as low as $75 \%$ by cooling at the trailing edge. The fluctuations were, on the other hand, significantly reduced. When the pulsations introduced in the coolant stream, the reduction in the pressure jump was still attained although it was not as pronounced as the steady blowing. However, the amplitudes fluctuating at pulsating frequency grown as compared to base line trace. The lowest values were achieved by the $190 \mathrm{kPa}$ blowing case possibly due to the stabilizing effect of the high cooling level. The medium and low pulsating blowing similarly effects the forcing over the blades. Nevertheless, the distinctive sinusoidal shape of pressure variation was only kept by the low blowing case.
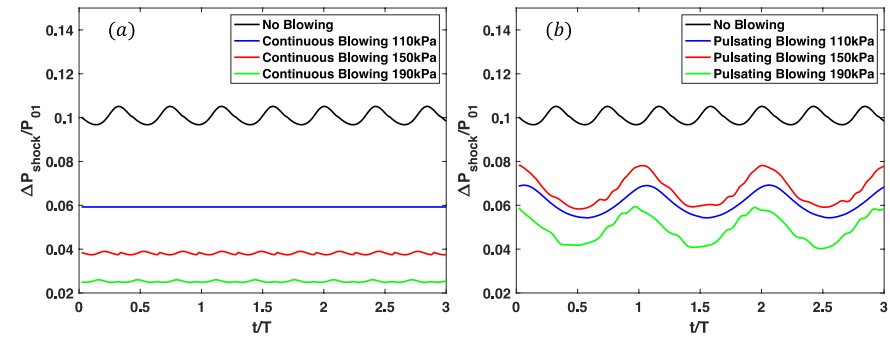

Fig. 9. Variation of static pressure jump across the shock with time for steady and pulsating blowing

\section{Conclusion}

Continuous and pulsating configurations of trailing edge coolant ejection with various blowing rates were investigated numerically. The effect of coolant purge to the base pressure levels, shock intensity values and rotor leading edge forcing characteristics are 
analysed. Filling the base region with high momentum cooling purge provided augmentation of base pressure which is accompanied with reduced shock intensities. The highest improvement on the modulation of shock wave was attained at value of $110 \mathrm{kPa}$ while further increase of purge mass flow into the base region resulted in marginal improvements. Although for pulsating cooling configurations employing high coolant flow rates possessed sub periodical oscillations over the general transient behaviour of pulsations, both cooling configurations provided promising results in terms of annihilating unsteady effects to lower amplitude oscillations without any influence on frequency compared to the no blowing case. Forcing analysis revealed significant improvements with continuous blowing configuration in terms of reducing amplitude of static pressure jump over the rotor plane while pulsating blowing induced low frequency oscillations with higher amplitude. Due to the implementation of pulsed blowing, variation of static pressure exerted on the leading edges of rotor blades elevated to higher values. Therefore, usage of high coolant flow rates for pulsating cooling applications, although reduction in the static pressure jump across the shock wave is observed to be directly proportional to the amount of coolant mass flow, might cause higher intensity pressure forces over the rotor blades, and thus, stress cycles which might lead to reduced lifetime of components.

\section{References}

1. F. Haselbach, Aero Engine Design - From State of the Art Turbofans Towards Innovative Architectures (Proceedings of VKI Lecture Series 2008-03), von Karman Institute, Brussels, Belgium, (2008).

2. J. D. Denton, The 1993 IGTI Scholar Lecture: Loss Mechanisms in Turbomachines. Journal of Turbomachinery, 115(4):621-656, (1989).

3. L. Xu, J. D. Denton, ASME 1990 International Gas Turbine and Aeroengine. Volume 1: Turbomachinery. Doi:10.1115/90-GT-3111, (1990).

4. G. Paniagua, T. Yasa, et al., Journal of propulsion and power, 24(4), (2008).

5. D. W. Maclachlan, D. M. Knowles, Fatigue and Fracture Engineering Material Science 25, Blackwell Science Ltd., Oxford, pp. 385-398, (2002).

6. S. Lavagnoli, T. Yasa, G. Paniagua, L. Castillon, S. Duni, ASME. J. Turbomach.; 134(1):011013-011013-13. doi:10.1115/1.4003241, (2011).

7. G. Paniagua, T. Yasa, A. de la Loma, L. Castillon, T. Coton, Journal of Propulsion and Power - J PROPUL POWER. 24. 722-731. 10.2514/1.34774, (2008).

8. J. Snoeck, C. H. Sieverding, M. Stanislas, Journal of Engineering for Power, 102(3):711-718, (1980).

9. L. Xu, J. D. Denton, Journal of Turbomachinery, 112(2):277-285, (1989).

10. J. J. Saavedra, G. Paniagua, B. H. Saracoglu, ASME. J. Turbomach.;139(6):061004061004-7. doi:10.1115/1.4035211, (2017).

11. D. Verrastro P. Adami A. de la Loma, G. Paniagua, Journal of Turbomachinery, 130(3):711-718, (2008).

12. C. H. Sieverding, Journal of Engineering for Power, 105(2):215-222, (1983).

13. F. J. Cunha, M. K. Chyu, Journal of Propulsion and Power, Vol. 22, No. 2, pp. 286300, (2006). 
14. O. Uzol, C. Camci, ASME. J. Turbomach. ;123(2):249-257. doi:10.1115/1.1351817, (2000).

15. W. Zhao, W. Luo, Q. Zhao, J. Xu, ASME. Turbo Expo: Power for Land, Sea, and Air, Volume 2B: Turbomachinery: V02BT38A005. doi:10.1115/GT2016-56131, (2016).

16. H. Rehder, ASME. J. Turbomach.;134(5):051029-051029-11. doi:10.1115/1.4004748, (2012).

17. B. H. Saracoglu, G. Paniagua, J. Sanchez, P. Rambaud, Journal of Computers and Fluids, 88:200209, (2013).

18. F. Martelli, G. Paniagua, B. H. Saracoglu, C. Bernardini, S. Salvadori, In V European Conference on Computational Fluid Dynamics ECCOMAS CFD 2010, Lisbon, Portugal, (2010).

19. B. H. Saracoglu, S. Salvadori, F. Tomasoni, S. Duni, T. Yasa, A. Miranda, G. Paniagua, Journal of Applied Thermal Engineering, 48:110, (2012).

20. D. C. Wilcox, DCW Industries, Inc., La Canada, CA, United States, NASA-CR191473, NAS 1.26:191473, DCW-R-35-03, DAAL03-89-C-0032; NASA ORDER L54880-C; RTOP 505-59-50-01, (1993)

21. N. Gourdain F. Duchaine T. Leonard, L. Y. M. Gicquel, Journal of Turbomachinery, 137, (2015).

22. B. O. Cakir, Active/Passive Flow Control Strategies for Base Pressure Control and Shock Modulation in Supersonic Confined Flows. VKI STP Report. (2017) 